\title{
Diseño Curricular Centrado en las Competencias que debe Adquirir el Estudiante del Grado en Derecho
}

\author{
María Goñi y Silvia Meseguer \\ Universidad Complutense de Madrid, Centro Universitario Villanueva, C/ Claudio Coello, 11, \\ 28001, Madrid-España (e-mail: mgoni@villanueva.edu; smeseguer@villanueva.edu)
}

\begin{abstract}
Resumen
Se determinan y analizan las competencias necesarias que debe adquirir un estudiante de Grado en Derecho para satisfacer el mercado laboral actual. Se realizó un estudio de campo entre profesionales del mundo del Derecho para comprobar la demanda del mercado laboral y conocer las competencias básicas y técnicas necesarias de los futuros profesionales. Se propone el modo de implantarlas para conseguir la renovación de las metodologías docentes. Como resultado se ha detectado la necesidad de utilizar herramientas docentes más modernas como el paso de la lección magistral a la expositiva, la aplicación de la metodología anglosajona a los casos prácticos, la elaboración de trabajos de investigación, el Practicum y la aplicación de las TIC's. En conclusión, las competencias de un graduado, la renovación de las metodologías docentes, y la demanda del mercado laboral, deben ponerse en estrecha relación para diseñar nuevos planes de estudios de Derecho.
\end{abstract}

Palabras clave: competencias, innovación docente, metodologías docentes, mercado laboral

\section{Curricular Design based upon the Skills to be Acquired by a Law Degree Student}

\begin{abstract}
The necessary skills that a student of a Law Degree should acquire to satisfy the current professional market are determined and analyzed. A field study among law professionals was done to determine the basic and technical skills that the labor market demands. A method for implementing them to achieve a renovation of the current teaching methods is proposed. As a result of the study, the necessity of using new teaching methods such as the change from the masterly lesson to a more practical one, the application of the anglo-saxon methodology to practical cases, the elaboration of research works, the Practicum and the use of TICs, have been detected. As a conclusion, the student skills along with the renewal of the teaching methods and the professional market have to be intimately put together to design new Law Study Planning.
\end{abstract}

Keywords: skills, teaching innovation, teaching methods, professional market 


\section{INTRODUCCION}

La nueva organización de las enseñanzas universitarias cumpliendo los criterios de convergencia europeos supone, además de un cualitativo cambio estructural, un significativo impulso en la renovación de las metodologías docentes que centran el objetivo en el proceso de aprendizaje del estudiante, en un marco que se extiende ahora a lo largo de la vida. Aprendizaje que, sin excluir el tradicional enfoque basado en los contenidos, se centra en la adquisición de competencias por los estudiantes, así como en los procedimientos para evaluar su adquisición. Las competencias, por tanto, son la referencia para estructurar los nuevos planes de estudio del Grado y servirán para diseñar los estudios de posgrado: Máster y doctorado.

Dejamos a un lado la definición de competencia que puede ser abordada desde distintas perspectivas (Riesco, 2008), pero nos interesa resaltar que cualquiera que sea la definición por la que optemos, todas se relacionan con la persona y con lo que ésta es capaz de lograr o la forma de abordar ciertas tareas con responsabilidad. Sin embargo, se debe dar un paso más. Las competencias están siempre ligadas al conocimiento puesto que no pueden desarrollarse sin el aprendizaje en algún campo o disciplina concreto y determinado, aunque no se limite a ellos (Perrenoud, 1999). En consecuencia, tres son los ámbitos de una competencia (Zabalza, 2005 Y Cabero, 2005). En primer lugar, el grado de conocimiento o nivel de dominio que se posee en relación a algo (dominar conceptos, teorías, clasificaciones); en segundo lugar, ejecutar la capacidad para utilizar el conocimiento en actuaciones prácticas (por ejemplo, desde el punto de vista jurídico, preparación de dictámenes e informes para instituciones públicas o privadas, redacción de contratos, etc.). Y tercero, presentar una actitud ética que implica unos valores necesarios para el ejercicio profesional, y con un estilo concreto de actuación (compromiso ético) (Riesco, 2008).

Y si, como hemos puesto de relieve, la competencia es la capacidad para enfrentarse a una tarea con cierta autonomía y responsabilidad, es necesario que se adquiera mediante el ejercicio de metodologías adecuadas a los estudios de Grado en cuestión. Pues bien, será necesario determinar qué competencias debe adquirir el estudiante y qué metodologías puede utilizar el profesor para enseñarlas y el alumno para aprenderlas. Respecto del primer aspecto debemos distinguir entre las competencias en genéricas y especificas.

Las competencias genéricas o transversales se definen como las destrezas que debe adquirir un estudiante y que son comunes a cualquier titulación. Las competencias genéricas situadas dentro de la sociedad del conocimiento gozan de una gran importancia y pueden ser comunes a diversas titulaciones. Así ocurre, por ejemplo, con la capacidad de aprender, tomar decisiones, etc. Se trata de una lista genérica, exhaustiva pero no taxativa, que tiene una función instrumental y que se adaptará para planificar títulos, materias, etc. El denominador común de las competencias genéricas es que se trata de competencias formales, en el sentido que se pueden adquirir a través de cualquier materia o contenido concreto (García, 2007). Y además, si el estudiante es el que debe adquirir estas competencias, sin duda, será necesaria su implicación para poder adquirirlas. Corresponderá al mercado laboral determinar las competencias; corresponderá al equipo rector de las universidades incluirlas en los planes de estudios pero en definitiva corresponderá al profesor planificar su asignatura conforme a ellas, y al estudiante implicarse en su desarrollo para que las pueda adquirir satisfactoriamente.

Las competencias, así entendidas, se convierten en el elemento básico del diseño curricular en distintos niveles: estructuran los nuevos planes de estudios de Grado, el diseño de las asignaturas específicas, y los nuevos métodos de evaluación. En consecuencia, hay que plantearse qué competencias debe adquirir un estudiante ante un Plan de Estudios determinado y, a continuación, definir qué competencias concretas debe adquirir en cada asignatura, que forman parte del todo. Estas competencias, ya lo hemos adelantado, deben hacer referencia a qué debe saber el alumno, qué debe saber hacer y qué actitudes debe tener para incorporarse al mercado profesional. Por otra parte, la definición de perfiles académicos y profesionales en las titulaciones está íntimamente ligada a la identificación y desarrollo de las competencias y destrezas y la manera de obtenerlas. La cuestión no hace referencia a sí debemos explicar más teoría o práctica, es decir, el número de horas dedicado a lecciones magistrales y el número de horas 
dedicadas a resolver supuestos prácticos. Se trata, más bien, de determinar qué competencias deben adquirir los estudiantes de una determinada asignatura y a partir de ahí estructurarla, a través de teoría, o práctica, o ambas a la vez.

De este modo, el profesor cuando elabora el plan docente de la asignatura debe responder a las preguntas que se plantea Aristimuño (Aristimuño, 2004): ¿cómo contribuye mi asignatura al logro de las competencias transversales y de las específicas? ¿qué competencias estoy logrando con los contenidos de mi asignatura?, o al revés ¿qué contenidos, dinámicas de aula y formas de evaluar son los más adecuados para lograrlas? La asignatura ha de contribuir al fomento y adquisición de determinadas competencias básicas o necesarias para el graduado, y todas ellas deben complementar el perfil competencial previamente definido. Consecuentemente, el desarrollo de las competencias exige la aplicación de nuevas metodologías, nuevas herramientas en la docencia. Según cuál sea la competencia que se pretenda obtener en cada asignatura, el profesor deberá utilizar una herramienta docente u otra -vgr. análisis de jurisprudencia, redacción de contratos, simulación de juicios-. El profesor ya no puede permanecer en la antigua "lección magistral", debe innovar, y debe fomentar el uso de estas herramientas alternativas, que se van a convertir en el eje básico de su docencia.

En este sentido, el Ministerio de Educación y Ciencia de España, en el Borrador de propuesta Directrices para la Elaboración de títulos Universitarios de Grado y Máster, Madrid, 2006, definía la relación entre las competencias y los nuevos recursos y metodologías docentes, de esta forma: "El plan de estudios conducente a la obtención de un título debe tener en el centro de sus objetivos la adquisición de competencias por parte de los estudiantes, ampliando, por tanto (aunque no excluyendo) el tradicional enfoque basado principalmente en contenidos y horas lectivas. Se deberá hacer énfasis en los métodos de aprendizaje de dichas competencias, así como en los procedimientos para evaluar su adquisición".

Y, en última instancia, se evaluarán junto a los conocimientos técnicos de la asignatura en cuestión, las competencias previamente definidas para esa asignatura; y en este sentido, no se trata sólo de adquirir conocimientos sino de saber qué se hace con ellos (Delgado, 2006 a). Y a pesar que otra idea nos podamos forjar, es evidente que no todas las competencias se desarrollarán en todas las asignaturas, ni en el mismo grado, por lo que será necesario planificar un reparto de competencias -y su evaluación- entre las diferentes asignaturas de un mismo Grado (Delgado, 2006 b).

Ahora bien, las competencias de un graduado deben ponerse en estrecha relación con el mercado laboral; esto es así, pues es una exigencia directa de la implantación del EEES. Distintos documentos ponen en relación las competencias con el ejercicio profesional del graduado, pues deben desarrollarse aquellas que faciliten al estudiante su incorporación en el mercado laboral (Ministerio de Educación y Ciencia de España, MEC), Borrador de propuesta Directrices para la elaboración de títulos Universitarios de Grado y Master, de 21 diciembre de 2006, apartado 21). De este modo, el Preámbulo del Real Decreto 55/2005 de 21 de enero señala que el objetivo de la enseñanza de grado es dar al estudiante una formación universitaria que aúne los conocimientos generales básicos, los conocimientos transversales relacionados con su formación integral "junto con los conocimientos y capacidades específicos orientados a su incorporación al ámbito laboral".

De igual forma el Borrador de propuesta Directrices para la elaboración de títulos Universitarios de Grado y Master, asegura que los nuevos títulos universitarios deben servir para la movilidad de los estudiantes y "deben preparar para el acceso al ejercicio profesional, es decir deben tener como objetivo la amplia empleabilidad de sus titulados". En términos muy parecidos se manifiesta la Comunicación de la Comisión de la Unión Europea (CUE, 2004), "Movilizar el capital intelectual de Europa: crear las condiciones necesarias para que las universidades puedan contribuir plenamente a la estrategia de Lisboa", cuando afirma que es necesario revisar los planes de estudios universitarios para "poder dar respuesta a las cambiantes necesidades de los mercados de trabajo", y continúa diciendo que "la integración de los titulados universitarios en la vida profesional, y por ende en la sociedad, es una de las principales responsabilidades de la enseñanza superior ante la sociedad". 


\section{LA APLICACIÓN A LOS ESTUDIOS DE DERECHO}

A continuación, con estas premisas, pretendemos abordar el caso concreto de los estudios de Grado en Derecho. Para ello, hemos tenido en consideración las competencias definidas en el Libro Blanco de Derecho (Agencia Nacional de Evaluación de la Calidad y Acreditación), que recoge las del Proyecto Tuning (2004), y las hemos completado con las obtenidas en el estudio que hemos llevado a cabo entre los profesionales del Derecho, con la finalidad de incorporar a las competencias más académicas aquellas que demanda el mercado laboral. Nos referimos al estudio de campo realizado por un grupo de profesores del Centro Universitario Villanueva, dentro del proyecto de investigación: "Análisis y elaboración de un catálogo de competencias y herramientas aplicables a las disciplinas de Ciencias Sociales y Jurídicas", y que se recoge en la obra colectiva "Enseñar Derecho en el siglo XXI", Thomson Aranzadi, Pamplona, 2009.

El estudio se planteó partiendo y recogiendo lo establecido en el Libro Blanco, y para el mismo, se elaboró un cuestionario con dos partes diferenciadas: en la primera se preguntaba sobre las competencias genéricas, y en la segunda sobre las competencias específicas que debe tener un graduado en Derecho. Al inicio del cuestionario se planteó un pregunta mas general, en la que se preguntaba al encuestado si consideraba que las personas empleadas en su empresa, licenciados en Derecho, han recibido o no la formación adecuada necesaria para desempeñar el trabajo asignado, y que se valorarse en un índice del 1 al 5 (mucho, bastante, algo, poco o muy poco).

A continuación se facilitaban un listado de 25 competencias genéricas, para que se valorarse su importancia para el desempeño del trabajo (en una escala de 1 a 4), y además, el nivel en que dicha competencia se había desarrollado en la Universidad hasta ahora, es decir, en la licenciatura tradicional de Derecho (también valorada de 1 a 4). En la segunda parte del cuestionario se pedía a los encuestados que indicaran hasta que punto es importante que un estudiante adquiera las distintas competencias específicas que se enumeraban -treinta en total-, tanto en Primer Ciclo como en Segundo Ciclo de la carrera de Derecho conforme al nuevo diseño de Planes de Estudio del EEES. En definitiva, con ese cuestionario se pretendía obtener como resultado qué competencias se consideraban más importantes para el ejercicio profesional, si hasta ahora se habían conseguido, y si consideraban acertado que se adquirieran, y por tanto se desarrollaran, a través de la nueva formación universitaria de Grado.

La encuesta iba dirigida a los empleadores de estudiantes de Derecho, y entre ellos se distinguieron los siguientes perfiles: a) Instituciones y Administración del Estado (Colegios profesionales: procuradores, abogados, notarios y registradores; Abogacía del Estado; Fiscalía; Tribunales (Supremo, Superiores de Justicia y de Instancia) b) Asesorías jurídicas de empresas y empresas c) Despachos profesionales de tamaño mediano o pequeño d) Despachos profesionales de gran tamaño. Nos interesaba conocer de primera mano, sobre todo, la opinión de la salida más frecuente, o natural de un graduado en Derecho: el ejercicio de la abogacía.

Pero, como ya hemos dicho, la base de nuestro estudio fueron las competencias y habilidades que el Libro Blanco del Título de Grado en Derecho de la ANECA, considera indispensable desarrollar a lo largo de la formación integral de los Graduados en Derecho. La enumeración es la siguiente:

-Tomar conciencia de la importancia del Derecho como sistema regulador de las relaciones sociales.

- Conseguir la percepción del carácter unitario del ordenamiento jurídico y de la necesaria visión interdisciplinaria de los problemas jurídicos.

- Capacidad para utilizar los principios y valores constitucionales como herramienta de trabajo en la interpretación del ordenamiento jurídico.

- Capacidad para el manejo de fuentes jurídicas (legales, jurisprudenciales y doctrinales).

- Desarrollo de la oratoria jurídica. Capacidad de expresarse apropiadamente ante un auditorio.

- Capacidad de leer e interpretar textos jurídicos.

- Capacidad de redactar escritos jurídicos. 
- Dominio de las técnicas informáticas en la obtención de la información jurídica (bases de datos de legislación, jurisprudencia, bibliografía).

- Capacidad de utilizar la red informática (Internet) en la obtención de la información y en la comunicación de datos.

- Adquisición de una conciencia crítica en el análisis del ordenamiento jurídico y desarrollo de la dialéctica jurídica.

- Adquisición de valores y principios éticos.

- Desarrollo de la capacidad de trabajar en equipo.

- Capacidad de negociación y conciliación.

- Conocimientos básicos de argumentación jurídica.

- Capacidad de creación y estructuración normativa.

- Comprensión y conocimiento de las principales instituciones públicas y privadas en su génesis y en su conjunto.

- Comprensión de las distintas formas de creación del derecho en su evolución histórica y en su realidad actual.

Estas competencias deben ponerse en relación con los resultados obtenidos del citado estudio de campo; destacamos como más interesantes los siguientes:

El primer dato relevante que se obtuvo es que, en su mayoría (un 75\%), los encuestados consideran que las personas licenciadas en Derecho que están trabajando con ellos, han recibido una formación bastante adecuada para el desempeño de su trabajo. Este dato es sorprendente si se pone en relación con las siguientes preguntas sobre competencias genéricas que, en general, los encuestados consideran que no se han desarrollado de forma suficiente en la Universidad, lo que nos hace concluir que al responder a la primera pregunta, se están teniendo en cuenta mas los conocimientos técnicos o teóricos (competencias específicas académicas) y no tanto las competencias genéricas, entendidas como las destrezas que debe adquirir un estudiante, sea cual sea su titulación.

En cuanto a las competencias genéricas que consideran más importantes para el ejercicio profesional, hay que decir que, en general, la mayoría de ellas son calificadas con la máxima puntuación, lo que indica que son bien valoradas y apreciadas por los empleadores. Sin embargo, su puntuación desciende si preguntamos si se han desarrollado suficientemente en la Universidad; luego, se detecta una carencia real en los estudiantes de Licenciatura de Derecho, pues aquellas competencias más deseadas no se han proporcionado suficientemente a través de la formación universitaria. Dato importante a la hora de planificar los nuevos planes de Grado en Derecho, para que fomenten dichas competencias.

Los resultados en cuanto a las competencias específicas, su importancia y si deben, y dónde contemplarse en los nuevos Planes de Estudios pueden resumirse de la siguiente forma: En general, se aprecia que es más importante que las competencias específicas de un estudiante de Derecho se adquieran en el segundo ciclo de la carrera que en el primero; es decir, que en ese segundo ciclo debe hacerse un especial esfuerzo para que el alumno obtenga dichas competencias, probablemente por la cercanía temporal con su salida al mercado laboral, que es donde realmente se le van a exigir. De esta forma, se constata que a grandes rasgos todas las competencias, hasta las menos valoradas, tienen una mayor consideración en el segundo ciclo que en el primero.

Por otra parte se aprecia que en el primer ciclo tienen una ligera mayor relevancia algunas de las competencias mas académicas (saber) o profesionales (saber hacer), pero estas últimas cuando se tratan de meros instrumentos o técnicas necesarias para poder seguir estudiando la carrera, por ejemplo: el manejo de fuentes jurídicas, el tomar conciencia del Derecho como sistema regulador de las relaciones sociales; conseguir la percepción del carácter unitario del ordenamiento jurídico, la comprensión de las distintas formas de creación del derecho en su evolución histórica y en su realidad actual; comprensión y conocimiento de las principales instituciones públicas y privadas en su génesis y en su conjunto. 
Aún y todo, muchas de las competencias se consideran que son importantes tanto en el primer como en el segundo ciclo, ya que coinciden en muchas ocasiones en sus valoraciones. Si aparecen como importantes en ambos ciclos, podemos decir, sin temor a equivocarnos, que esas competencias bien valoradas en ambos ciclos, son las competencias específicas que más se aprecian por los empleadores y que por tanto se consideran imprescindibles en un estudiante de Grado en Derecho y que, en consecuencia, deberán incluirse en los Nuevos Planes de Estudios. Uniendo y analizando conjuntamente los resultados obtenidos respecto a las competencias genéricas y a las específicas, considerando que algunas de las específicas no son sino concreciones de aquellas mas generales, y sumando las cifras de valoración de cada una, podemos concluir que las competencias (genéricas y específicas) mejor valoradas, y que, por tanto, deben tenerse presentes a la hora de estructurar el Grado en Derecho, para que los estudiantes puedan adquirirlas, y en consecuencia, acceder con mas facilidad al mercado laboral, deben ser:

Capacidad de análisis y síntesis.

Capacidad de comunicación oral: oratoria y retórica -jurídica-.

Capacidad de negociación.

Capacidad de redacción -de textos jurídicos-.

Capacidad de argumentación y convicción.

Compromiso ético.

Capacidad de resolución de problemas, aplicando los conocimientos a la práctica.

Definidas estas competencias y cotejándolas con las del Libro Blanco, llegamos a la conclusión de que un graduado en Derecho, como mínimo, y fundamental debe desarrollar y obtener en su caso las siguientes competencias mas valoradas en ambos estudios: las siete que han salido en nuestro estudio que son a su vez, siete de las que establece el Libro Blanco y además otras 3 competencias específicas, menos valoradas, pero sí importantes:

i) La toma de conciencia de la importancia del Derecho como sistema regulador de las relaciones sociales.

ii) La capacidad para utilizar los principios y valores constitucionales como herramienta de trabajo en la interpretación del ordenamiento jurídico.

iii) La adquisición de una conciencia crítica en el análisis del ordenamiento jurídico y desarrollo de la dialéctica jurídica.

No podemos desconocer otras competencias, como el conocimiento de una lengua extranjera, los conocimientos de informática relativos al ámbito de estudio, o la capacidad de trabajo en equipo, que, por ser comunes a todas las titulaciones, no aparecen reflejadas con tal fuerza en nuestro estudio, sin que, por ello, puedan minusvalorarse. Estas peculiares competencias deben servir ahora de estructura del nuevo grado en Derecho, en los niveles señalados: Plan de estudios, asignaturas/nuevas metodologías, evaluación e implicación del alumno en todo el proceso.

\section{Estructura del Plan de estudios}

Atendiendo a las competencias que deben adquirir los estudiantes de Derecho, se establecen unas pautas que deben inspirar la configuración de los estudios de Grado en las Facultades de Derecho. Así, se hace referencia a una troncalidad que constituye el $65 \%$ de la estructura general del título, y se le asignan 156 créditos ECTS frente a los 240 totales que corresponden al título. El $30 \%-35 \%$ restante se configura a través de asignaturas optativas que les permite incluir las dobles titulaciones.

Quizás una de las conclusiones más interesantes obtenidas del análisis realizado por el Libro Blanco es que en el Grado en Derecho, sin excluir algunas especificidades, las destrezas y habilidades exigibles presentan un alto grado de homogeneidad en gran parte de las materias, lo que conduce a hablar de transversalidad de las competencias jurídicas y, en consecuencia, a agrupar las materias en función de una mayor o menor afinidad. 
Y esta afinidad de las materias jurídicas se concreta por su pertenencia a los ámbitos del Derecho Público o Derecho Privado, siendo necesaria la subdivisión del bloque de Derecho Público en dos subbloques, Derecho Público I y Derecho Público II, por el creciente desarrollo que ha tenido en los últimos tiempos. Además, se incorpora un tercer bloque de carácter formativo al que se denomina "Ciencias Jurídicas Básicas". Y el "Practicum" que agruparía las actividades de carácter instrumental relacionadas con los cuatro bloques de materias anteriores (Derecho Público I y II, Derecho Privado y Ciencias Jurídicas Básicas) (Libro Blanco de Derecho, ANECA).

Respecto del Practicum, los Graduados en Derecho consideraron insuficiente la formación práctica y mencionaron la necesidad, en la mayoría de los casos, de completar la formación con postgrados o estudios de segundo ciclo. No se dudó en hacer una recomendación final de indudable trascendencia como es "la de proponer un fuerte reforzamiento de las clases prácticas a desarrollar en cada una de las asignaturas, y en especial en la potenciación del Practicum, que los alumnos deberán realizar fuera de la universidad, en diferentes instituciones y organismos jurídicos colaboradores con ella" (Libro Blanco de Derecho, ANECA).

\section{Configuración de asignaturas}

Es un hecho evidente que la aplicación de estas competencias a los planes de estudios requieren la renovación de las metodologías docentes: el profesor deberá renovar los sistemas tradicionales de enseñanza de la asignatura que imparte en el plan de estudios. Es decir, deberá plantear su materia determinando las competencias genéricas y específicas que debe adquirir el alumno. A continuación, deberá plasmarlas en su guía docente, eligiendo las herramientas necesarias para facilitarlas y elaborando los materiales docentes necesarios para ello. Entre las herramientas que puede utilizar el profesor para conseguir los objetivos determinados en su planificación docente, destacamos la lección magistral, hoy transformada en lección expositiva, que debe combinarse con las herramientas alternativas al aula -tales como seminarios, asistencia a juicios, etc.-, todas ellas dirigidas a enseñar a los alumnos a reflexionar desde una perspectiva analítica y sintética. Asimismo, destacan como herramientas específicas aplicables a los estudios de Grado en Derecho, la redacción de textos legales, el análisis de jurisprudencia y la elaboración de un trabajo de investigación. Se trata de herramientas clave para el futuro graduado en Derecho porque a partir de ahora será necesario, más que nunca, en orden a superar cada uno de los ciclos en que se estructuran los estudios, la elaboración de un trabajo de investigación y aprender la metodología y formalidades necesarias para resolver los problemas jurídicos que se le planteen en el desempeño profesional.

Por otra parte, un planteamiento abierto y dinámico de la enseñanza del Derecho conlleva la aplicación de la metodología anglosajona a los casos prácticos, a través de la cual, el alumno será capaz de aplicar a la práctica los conocimientos adquiridos a nivel teórico, que le servirán como entrenamiento para su futuro profesional. E, igualmente, permite la aplicación de otras herramientas novedosas como el estudio de la comparación de sistemas jurídicos, la simulación de juicios, el uso de las TIC's y los entornos virtuales de aprendizaje (Monterroso, 2009), así como la oratoria jurídica o la capacidad de negociación o mediación que se convierten en herramientas que el alumno debe saber manejar para el ejercicio de su profesión. Por tanto, llegados a este punto será necesario hacer un esfuerzo para conectar cada una de las competencias y la forma de obtenerla dentro de una asignatura concreta enmarcada en plan de estudios determinado. Así y sin ánimo exhaustivo, para desarrollar las competencias clave determinadas, el profesor podrá utilizar las siguientes herramientas:

\section{Capacidad de análisis y síntesis}

Como herramientas que desarrollan estas competencias podemos mencionar: análisis de jurisprudencia, elaboración de un trabajo de investigación, resolución de casos prácticos, interpretación de textos legales.

Capacidad de comunicación oral: oratoria y retórica

Un estudiante de Grado en Derecho debe adquirir paulatinamente esta competencia a lo largo de su formación universitaria, lo cual implica que, una vez mas, el alumno debe ser protagonista en su formación, participando en debates, exposiciones orales, simulaciones de juicios. 


\section{Capacidad de negociación}

La negociación, además de una competencia que se debe adquirir, es en sí misma una herramienta, y debe constituirse o en una asignatura independiente, o transversal en otras materias.

\section{Capacidad de redacción de textos jurídicos}

Las herramientas para fomentar esta competencia son la propia redacción de textos legales por los alumnos en clase, la elaboración de un trabajo de investigación o un análisis jurisprudencial, la resolución de casos prácticos, y también, por qué no, la realización de exámenes escritos que van acostumbrando al estudiante de Derecho a expresarse correctamente en este peculiar ámbito.

\section{Capacidad de argumentación y convicción}

Es fundamental desarrollar esta competencia, mediante la elaboración de trabajos de investigación en los que se exponen y argumentan tesis, la resolución de casos prácticos que busca sobre todo el razonar y argumentar posturas, y a través de las simulaciones de juicios, en los que, primero de forma escrita y luego en exposición oral, deben convencer de sus posturas previamente razonadas y argumentadas.

\section{Compromiso ético}

El compromiso ético debe enseñarse en la Universidad, no como asignatura. sino impregnando cada una de las ramas del saber y en cada actuación y enseñanza impartida.

Capacidad de resolución de problemas, aplicando los conocimientos a la práctica. Probablemente un buen jurista se caracteriza por "solucionar problemas", "casos", y por tanto, esta competencia debe tratar de desarrollarse especialmente; para ello, es necesario la resolución de casos prácticos, tanto reales, que reflejen un problema de la realidad, como los de "laboratorio" propuestos por el profesor con la intención de guiar al alumno a la búsqueda de la solución que él pretende. También la simulación de juicios fomenta esta competencia, así como las técnicas de negociación, pues la negociación es uno de los procedimientos más relevantes para la resolución de conflictos.

\section{Conocimiento de una segunda lengua}

Como hemos dicho esta competencia no aparece reflejada como una de las más relevantes en el Libro Blanco, pero si se considera básica en el entorno profesional. El desarrollo de esta competencia se conseguirá impartiendo seminarios o asignaturas específicas a través de la comparación de sistemas jurídicos, impartidos en otro idioma.

\section{Evaluación}

Las exigencias docentes de los criterios de convergencia europea traen consigo una reforma de los métodos de evaluación. A partir de ahora se evaluará en función de las competencias que debe adquirir el graduado. Por ello, surgen nuevos métodos de evaluación, se evalúan nuevas actividades, y se inclina el peso de la misma hacia el propio trabajo del alumno -saber hacer-, más que hacia su conocimientos -saber-, estableciéndose un sistema de evaluación continúa que pondere el uso de todas las herramientas citadas.

Siguiendo a López Álvarez (López, 2009), si al aprendizaje se le define como proceso, parece acertado indicar que su evaluación también debe serlo, sobre todo si, como suele ser habitual, el proceso del aprendizaje dura varios meses. No sería prudente esperar al final, cuando ya no hay remedio, para evaluar si el aprendizaje está resultando adecuado. Por numerosos motivos, entendemos la evaluación "como un proceso que se inicia al principio de curso cuando profesor y alumnos entran en contacto, negocian los elementos del programa, y se asumen niveles de exigencias y trabajo individual y grupal" (Marcelo, 2001). De este modo, hay que evitar la disociación entre la labor formativa y la evaluadora, y hay que ser capaz de diseñar un sistema de evaluación coherente con el sistema docente adoptado, y no dejarlo todo para un examen final, a veces insuficiente y desproporcionado con lo explicado durante el curso, con el riego que esto supone; pues puede fomentar únicamente el aprendizaje estratégico y puede distorsionar la 
medición realizada por la evaluación (Bain, 2007). En definitiva, la prueba evaluadora debe medir conocimientos y capacidades y la mejor manera de asegurar que se mantiene el equilibrio seguido durante el aprendizaje es utilizar una prueba similar a las realizadas durante el desarrollo del curso (Delgado, Las competencias fundamentales, 2006 y Goñi, 2005), y por eso abogamos por un sistema de evaluación continúa como el más adecuado.

\section{Implicación del alumno en el proceso de aprendizaje}

Sin embargo, también este método tiene sus problemas, pues puede exigir un ritmo de trabajo diferente al que están acostumbrados los alumnos, que puede perjudicar su rendimiento en el resto de asignaturas; lo que conlleva a una necesaria labor de coordinación entre los profesores implicados en la docencia de un mismo curso. Por tanto, es necesario una buena planificación de los trabajos y pruebas que van a componer la evaluación continua, sin olvidar el examen final acumulativo, que consideramos debe seguir teniendo su peso específico en la nota final.

La asistencia a clase se perfila como nuevo elemento relevante en la evaluación, si bien, en aquellos casos de alumnos no presenciales, habrá que sustituir la presencialidad por otras herramientas, probablemente on line, que impliquen para el alumno la misma carga de trabajo necesaria para superar la asignatura.

Por otra parte, no se puede olvidar el papel del alumno en este nuevo proceso; es necesaria su implicación en este reto, para compensar la imagen de exceso de trabajo que el nuevo sistema le exige, con una mejor preparación para afrontar la incorporación en el mercado laboral. El alumno, por su parte, debe estar preparado para el cambio. En efecto, ahora el estudiante es la parte integrante del aprendizaje y no se puede limitar a ser un mero receptor pasivo. La implicación del alumno será mucho mayor a través de la realización de casos prácticos, actividades fuera del aula, trabajos de investigación, seminarios, etc., que le permitirán el aprendizaje a lo largo de toda la vida (long life learning) porque la educación es, como señalan los criterios de convergencia del EEES, continua y permanente. Se considera que los conocimientos son cambiantes, y debe proporcionarse al alumno las competencias y habilidades necesarias para saber afrontar estos cambios.

\section{CONCLUSIONES}

Las competencias, también las demandas por el mercado profesional, son la clave de la planificación docente, a tres niveles diferentes: en el diseño de los planes de estudios que se deben adecuar a las exigencias del mercado laboral; en la configuración de las asignaturas específicas; y en consecuencia, en la aplicación de nuevos métodos de evaluación que integren los conocimientos técnicos con las competencias y habilidades del estudiante.

Por eso, a la hora de abordar un plan de estudios, hay que definir, primero, las competencias que se quieren conseguir, en el sentido de que debe quedar claro lo que el alumno debe saber, lo que debe saber hacer, y las actitudes y aptitudes que le van a exigir.

Ahora bien, determinadas las competencias, y el plan de estudios consecuente a las mismas, es necesario estudiar cómo desarrollar su potencialidad. En concreto, qué herramientas y métodos de evaluación se deben utilizar para conseguir de forma eficaz las competencias o destrezas previamente señaladas.

Por lo tanto, y como conclusión final, hay que renovar las metodologías docentes partiendo de las competencias concretas de cada grado universitario. Las herramientas de que dispone para ello son variadas, pero todas ellas exigen un cambio de mentalidad y un esfuerzo añadido de trabajo al profesor, sin perder de vista, claro está, el objetivo que se pretende en la planificación por competencias que es precisamente centrar el objetivo en el proceso de aprendizaje en el estudiante, en un marco que se extiende ahora a lo largo de la vida. 


\section{REFERENCIAS}

Agencia Nacional de Evaluación de la Calidad y Acreditación, Libro Blanco, Título de Grado Derecho, desde http://www.aneca.es/activin/docs/libroblanco_derecho_def.pdf.

Aristimuño, A., Las competencias en la educación superior: ¿demonio u oportunidad?. Departamento de Educación, Universidad Católica de Uruguay, Uruguay,(2004).

Bain, K., Lo que hacen los mejores profesores ( $2^{\mathrm{a}} \mathrm{Ed}$ ), Universitat de Valencia, Valencia, España, (2007).

Cabero, J., Formación del profesorado universitario en estrategias metodológicas para la incorporación del aprendizaje en red en el Espacio Europeo de Educación Superior, Programa de Estudios y Análisis, Ministerio de Educación y Ciencia, Madrid, España, (2005).

CUE, "Movilizar el capital intelectual de Europa: crear las condiciones necesarias para que las universidades puedan contribuir plenamente a la estrategia de Lisboa", acceso 25 nov 2008, www.crue.org/export/sites/Crue/procbolonia/documentos/antecedentes/8._Movilizar_el_capital_int electual.pdf., Comisión de la Unión Europea, p. 6. Bruselas, (2004).

Delgado, A.M., El enfoque de la evaluación continua por competencias, en Enseñar Derecho en la red. Un paso adelante en la construcción del Espacio Europeo de Educación Superior, Bosch Editor, Barcelona, España, (2006).

Delgado, A.M., Las competencias fundamentales, en Evaluación de las competencias en el Espacio Europeo de Educación Superior: una experiencia desde el Derecho y la Ciencia política, Ed. Bosch, pp. 47-48, Barcelona, España, (2006).

García, J., Las competencias y la nueva organización de la enseñanza, en La Licenciatura en Derecho en el contexto de la convergencia europea, (Coord. María Reyes León Benítez), Ed. Tirant Lo Blanch, pp. 172-173, Valencia, España, (2007).

Goñi, J.M., El Espacio Europeo de Educación Superior, un reto para la Universidad. Barcelona, Ed. Octaedro/ICE Universidad de Barcelona, Barcelona, España, (2005).

López, L. F., Métodos de evaluación, en Enseñar Derecho en el Siglo XXI. Una guía práctica sobre el Grado en Derecho, Thomson Aranzadi, pp.351-367, Pamplona, España, (2009).

Marcelo, C., El proyecto docente: una ocasión para aprender, en García -Valcarcel MuñozRepiso, A. (coord.), Didáctica universitaria, La Muralla, Madrid, España, (2001).

Ministerio de Educación y Ciencia de España (MEC), Borrador de propuesta Directrices para la elaboración de títulos Universitarios de Grado y Master, (de 21 diciembre de 2006, apartado 21), (2001).

Monterroso, E., Los entornos virtuales de aprendizaje: una aplicación práctica, en Enseñar Derecho en el Siglo XX. Una guía práctica sobre el Grado en Derecho, Thomson Aranzadi, pp. 317-330, Pamplona, España, (2009).

OCDE-DESECO, Definition and Selection of Competencies: Theoretical and Conceptual

Foundations. Paris. Recuperado a 25 noviembre 2008, desde

www.deseco.admin.ch/bfs/deseco/en/index/03/02. parys78532.downloadlist, (2005).

Perrenoud, P., Construir competencias desde la escuela, Dolmen, Santiago de Chile, Chile, (1997).

Proyecto Tuning, desde http://tuning.unideusto.org/tuningeu/, (2004).

Riesgo, M., El enfoque por competencias en el EEES y sus implicaciones en la enseñanza y el aprendizaje, Tendencias pedagógicas, 13, pp. 79-105, (2008).

Rodríguez- Arana, J. y Palomino, R., Enseñar Derecho en el siglo XXI. Una guía práctica sobre el Grado en Derecho, Thomson Aranzadi, Pamplona, España, (2009).

Zabalza, M., Guía para la planificación didáctica de la docencia universitaria en el marco del EEES, Universidad de Santiago de Compostela, Santiago de Compostela, España, (2005). 